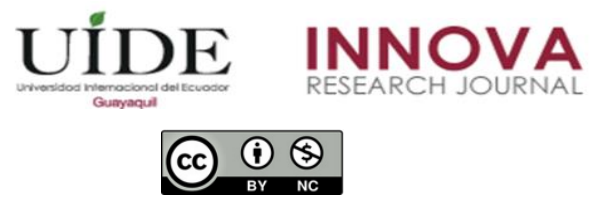

INNOVA Research Journal, ISSN 2477-9024

(Septiembre-Diciembre 2020). Vol. 5, No.3 pp. 245-261

DOI: https://doi.org/10.33890/innova.v5.n3.2020.1364

URL: http://revistas.uide.edu.ec/index.php/innova/index

Correo: innova@uide.edu.ec

\title{
Evaluación de los Estilos de Vida y Valores de los Turistas que visitan la Amazonía Ecuatoriana
}

\section{Evaluation of the Lifestyles and Values of Tourists visiting the Ecuadorian Amazon}

Luis Oswaldo Manosalvas Vaca

https://orcid.org/0000-0002-4659-8090

Carlos Aníbal Manosalvas Vaca

https://orcid.org/0000-0002-7521-069X

María Germania Gamboa Rios

https://orcid.org/0000-0002-0427-9016

Universidad Estatal Amazónica, Ecuador

Viviana Catalina Solís Morejón

https://orcid.org/0000-0002-5961-7406

Escuela Superior Politécnica del Chimborazo, Ecuador

Autor por correspondencia: 1manosalvas@uea.edu.ec; cmanosalvas@uea.edu.ec; viviana.solis@espoch.edu.ec; mgamboa@uea.edu.ec

Fecha de recepción: 04 de marzo del 2020 - Fecha de aceptación: 03 de julio del 2020

\section{Resumen}

La investigación analiza los valores y estilos de vida de los turistas que visitan la Amazonía ecuatoriana, y para este caso específico se evaluó una muestra de turistas que visitaron la ciudad de Puyo, provincia de Pastaza, con respecto a los diferentes atractivos turísticos, lugares de alojamiento y restauración, la evaluación se desarrolló mediante la aplicación de instrumentos de medición basado en el método LATIR de estilos de vida propuesto por el investigador Rolando Arellano y el método VALS II (values and life styles). La metodología fue con un enfoque cuantitativo, descriptivo y transversal mediante la aplicación de encuestas válidas y confiables usadas en varias investigaciones en otros contextos y regiones, con ello, se pudo establecer el perfil de los clientes para cada uno de los segmentos identificados, los resultados indicaron que el estilo de vida del turista que visita la región corresponde a Progresistas, Modernas y Sofisticados, con respecto a los valores, la dimensión que predomina es el turista entusiasta, las otras dimensiones arrojaron valores poco favorables y desfavorables, lo cual permite plantear estrategias para mejorar el turismo en la región.

Palabras clave: estilo de vida; valores; segmentación; turismo; Amazonía

\section{Abstract}

The research analyzes the values and lifestyles of tourists visiting the Ecuadorian Amazon, and for this specific case a sample of tourists who visited the city of Puyo, Pastaza province, was evaluated 
with respect to the different tourist attractions, places of accommodation and restoration, the evaluation was developed by applying measurement instruments based on the LATIR method of lifestyles proposed by the researcher Rolando Arellano and the VALS II method (values and life styles). The methodology was with a quantitative, descriptive and cross-sectional approach through the application of valid and reliable surveys used in various investigations in other contexts and regions, with this, it was possible to establish the profile of clients for each of the identified segments, the results indicated that the lifestyle of the tourist who visits the region corresponds to Progressive, Modern and Sophisticated, Regarding values, the predominant dimension is the enthusiastic tourist, the other dimensions they showed unfavorable and unfavorable values, which allows to propose strategies to improve tourism in the region.

Key words: lifestyle; values; segmentation; tourism; amazon

\section{Introducción}

La investigación tuvo como objetivo analizar los estilos de vida de los turistas que visitan la Amazonía ecuatoriana y generar estrategias que permitan mejorar la oferta turística mediante los resultados obtenidos, para desarrollar la investigación se identificó una segmentación de las personas que visitan los atractivos turísticos, restaurantes y alojamiento en la región amazónica del Ecuador, específicamente se analizó la dinámica turística de la ciudad de Puyo, Provincia de Pastaza y con ello se pudo identificar el tipo de cliente que visita la ciudad, con los resultados obtenidos, los prestadores de servicios turísticos pueden ofrecer un producto segmentado y dirigido a los diferentes estilos de vida identificado, al momento de conocer el perfil facilita un nuevo método de publicidad y mercadeo a los segmentos detallados, para mantener la competitividad en el sector turístico actual se debe estar al día con las nuevas tendencias que el visitante busca de acuerdo a sus necesidades.

Para realizar la valoración se utilizaron instrumentos de medición basados en el método LATIR y VALS II, siendo estas herramientas ventajosas para medir las actitudes de los consumidores, en las diferentes preguntas que se presentó en las encuestas, de la misma manera, se pudo identificar la situación demográfica, los valores, las actitudes y las variables de los estilos de vida de cada uno de los encuestados (Salgado \& Caremena, 2015)

Es importante conocer los diferentes estilos de vida dentro de Latinoamérica con la finalidad de conocer al consumidor; su comportamiento, su manera de pensar, actuar y querer a la hora de escoger un producto o servicio, a través del análisis las empresas prestadoras de servicio pueden segmentar a su consumidor de forma directa (Arellano, 2017). Según la Cámara de Turismo provincial, la ciudad de Puyo no ofrece diversidad de productos y servicios segmentados para los turistas nacionales y extranjeras de los diferentes estilos de vida que visitan, es por ello que fue necesario saber si la actividad turística que promociona dentro y fuera de la ciudad influye a los visitantes al momento de elegir visitar esta ciudad. 


\section{Marco Teórico}

\section{Turismo a nivel mundial}

En la última década el turismo a nivel mundial ha ido creciendo de manera paulatina convirtiéndose de esta manera en un papel importante para el desarrollo de la población a nivel mundial, además siendo una de las actividades más dinámicas donde el visitante puede disfrutar una variedad de productos y servicios de cada uno de los países visitados compartiendo experiencia con las diferentes culturas, al mismo tiempo generando divisas económicas, tasas de empleo, mejoramiento de las infraestructuras y sobre todo tener una mejor vida, asimismo generando la concientización de la población para el cuidado del medio ambiente (Nieto, Róman, Bonillo, \& Paulova, 2016)

\section{El turismo en el Ecuador}

La importancia del turismo en el Ecuador es indispensable ya que genera aportes económicos, fuentes de trabajo, diversifica las actividades turísticas, además el turismo internacional aporta divisas económicas con ello mejorando el estilo de vida de las poblaciones del área rural y urbana, es así que el Ecuador posee 'Los cuatro mundos' Región Insular, Costa, Andina y la Amazónica manteniendo un crecimiento importante a nivel mundial, en el sector de servicios las entidades públicas han jugado un papel importante realizando promociones, nuevas campañas y programas con la finalidad de aprovechar cada una de las riquezas que cuenta el país, ofreciendo al turista diversidad de actividades a realizar en cada de una de las regiones visitadas (Castillo, Martínez, \& Vazquez, 2015)

\section{El turismo en la Región Amazónica}

El turismo en la región Amazónica ha tomado relevancia en los últimos tiempos gracias a la promoción turística que ha realizado las autoridades competentes, asimismo esta región cuenta con una gran diversidad de flora y fauna endémica que posee, donde el turista nacional o internacional puede interactuar de manera directa con la naturaleza en su estado puro, además puede visitar diferentes centros turísticos comunitarios y así poder conocer las costumbres y tradiciones de las nacionalidades que posee cada una de las provincias, la provincia de Pastaza es la más grande del Ecuador, con su capital Puyo que está rodeada con una vegetación extraordinaria, volcanes, ríos, cascadas y en ellas habita las diferentes culturas milenarias ejemplo; Kichwa, Shuar, Achuar, Andoas, Zapara, entre otros (López, Espíndola, Calles, \& Ulloa, 2013)

\section{El turismo en la Provincia de Pastaza}

La provincia de Pastaza con su capital Puyo, perteneciente a la región Amazónica conocida como la 'Ciudad de Canela', cuenta con diferentes atractivos turísticos que se está potenciando con el pasar de los años, gracias a la existencia de tres corredores de conexión interprovincial hacia la ciudad (Puyo - Baños, Puyo - Tena, Puyo - Macas), la mayor atracción de los turistas que visitan la ciudad son el Boayaku Puyo, Barrio Obrero, Parque Etnobotánica Omaere, Jardín Botánico de las Orquídeas, Paseo de los Monos, Refugio de Vida Silvestre 
Yanacocha entre otros, cabe recalcar que en la provincia de Pastaza viven 7 nacionalidades que hacen único a la provincia a nivel nacional (Reyes \& Ortega, 2013)

\section{La segmentación por estilos de vida en el turismo}

Según Adler (1929), los estilos de vida se definen como las metas que las personas trazan y los medios que utilizan para poder alcanzarlos, además está relacionada con el marketing en el aspecto de la publicidad asimismo la segmentación del cliente por estilos de vida divide a grupos sociales según sus estatus económicos en grupos homogéneos con el fin de llegar al consumidor con estrategias de marketing específicamente enfocadas a cada uno de los estilos de vida, ya que esto enfoca directamente a los hábitos de consumo, entre ellas tenemos; los sofisticadas, progresistas, modernas, formales, conservadoras y los austeros, de esta manera busca satisfacer las necesidades de los clientes y a la vez cumplir los objetivos planteados por las empresas (Arellano, 2017).

Los estilos de vida y la segmentación de mercados, tuvo comienzos en los años 70 identificándose como las distintas maneras de vivir de las personas en una localidad esto permitiendo distinguirlos de otros grupos sociales siendo esto por gustos, situación demográfica, estatus económico, nivel de educación entre otros, es por ello que existe la necesidad de segmentar a las personas según sus estilos de vida y deseos de grupos identificados (Lazer, 1963)

La metodología de los estilos de vida con respecto en las investigaciones acerca de la motivación, está relacionada con las variables de psicología racional, concreta y comportamental permitiendo tener una visión completa del consumidor, por lo general las personas adquieren un estilo de vida dependiendo de los grupos dominantes donde vive o más bien se transforman para pertenecer a esa sociedad, se identifica los diferentes estilos de vida de acuerdo a su hábitos, su manera de vivir día tras día, ocupación laboral, diversión, su actitud y su manera de comportarse con los demás, su forma de gastar cuando acude a centros comerciales (Baudrillard, 1970)

La industria turística con el pasar del tiempo va teniendo protagonismo ya que existe mayor concientización por parte de los visitantes y prestadores de servicios en aspectos medioambientales teniendo un mayor énfasis entre el turismo, naturaleza, cultura y conservación, gracias aquello ha existido cambios en el consumidor modificando sus gustos y preferencias al momento de visitar, es por ello que es indispensable conocer a los turistas ofreciendo nuevos productos o servicios que vayan acorde a las nuevas tendencias que se están presentando en el medio turístico (Cordente, 2011)

Es importante poder identificar el perfil del turista que visita una localidad, esto se puede analizar gracias a los tipos de segmentación que existe, siendo importante conocer el factor demográfico con ello su género, edad, estado civil, nivel de estudios, su ingreso mensual o anual entre otros, asimismo se puede analizar las características psicodemográficas donde se puede identificar acerca de su personalidad, sus pensamientos, la manera de actuar, su conducta frente a los demás, así como conocer su estilo de vida y valores, con la finalidad de ofrecer los mejores productos o servicios al momento que realicen sus viajes y superar sus expectativas donde tengan una experiencia agradable (Kotler \& Keller, 2012) 
Además, el estilo de vida da énfasis en la forma de vida de un individuo o un grupo poblacional en las características personales, aspectos socioculturales y patrones del modo de su comportamiento, a su vez en estudios relacionados con el tema se enfocan en el aspecto sociodemográfico de la población y más bien no se da la importancia debida al aspecto psicográfico del turista que visita, se debe tener en cuenta que los turistas vienen a probar nuevas expectativas y experiencias de acuerdo a su estilo de vida (Campo, Beltran, \& Puello, 2017)

Las empresas turísticas que ofrecen servicios de; alojamiento, restauración, transporte y visita a atractivos turísticos han visto la necesidad de crear varias tipologías con productos diferenciados, de esta manera brindando a la demanda segmentada un producto o servicio propicio de acuerdo a su estilo de vida, ya que en la actualidad los visitantes están informados atreves de redes sociales, televisión, revistas y periódicos por ende son exigentes a la hora de recibir el producto o servicio por consiguiente las empresas deben ofrecer un servicio más personalizado (Fernandez, Blanco, \& Martinez, 2013)

Es necesario conocer los diferentes estilos de vida que existen a nivel de Latinoamérica ya que en los últimos años ha existido cambios extraordinarios, en crecimiento económico, estatus social, nivel de estudio, desarrollo de las empresas públicas y privadas, las exportaciones y los cambios políticos (Angelucci, Cañoto, \& Hernández, 2017)

\section{La psicografía en el turismo}

El origen del término psicográfico se determina para identificar personas por sus apariencias y en la actualidad se usa como un método de segmentación, de esta manera, surge por primera vez donde los investigadores empezaron a estudiar las motivaciones de compra de cada uno de las personas dentro un mercado, con la aparición de las computadoras la tabulación de las encuestas se volvió más fácil y con ellos se obtenía resultados matemáticos, porcentajes, promedios entre otros fiables; con el pasar de los años el termino psicográfico ha tenido una gran evolución en diferentes industrias incluyendo al turismo, dividiendo a los consumidores en diferentes grupos con diversos aspectos personales (Canovas \& Opazo, 2005)

Las variables intangibles pueden medirse mediante aspectos psicográficos, entre ellas podemos encontrar los estilos de vida, las actividades que realizan a diario, interés, sus opiniones, las motivaciones de compra o de sus viajes, su personalidad y actitudes, estudiando así los factores psicológicos, sociológicos y antropológicos, de esta manera se puede segmentar mejor el mercado objetivo siendo esta una alternativa de crear o renovar los productos o servicios con la finalidad que los prestadores de servicios turísticos se adapten mejor al mercado que se están enfocando (Boote, 1982)

Al momento de realizar la segmentación en base psicográfica del turista se divide en tres variables los cuales son (Ciribeli \& Miquelito, 2015)

- Personalidad: es un grupo de características psicológicas, que determina la manera de comportarse del turista al momento de viajar.

- Actitud: es la reacción del turista frente a los productos o servicios turísticos ofrecidos en el lugar visitado. 
- Estilo de vida: está vinculado como un visitante actúa frente a la sociedad, esto se conoce como un patrón de consumo del visitante, por ende, muestra sus valores, preferencias personales, el dinero que gasta, en que lo gasta y cuánto tiempo visita cierto lugar.

Dentro de las características psicográficas de un turista, el comportamiento que desempeña es importante ya que puede adoptar varios tipos de comportamiento al momento de visitar, teniendo entre ellas preferencias por ciertos lugares como; museos, iglesias, parques temáticos, restaurantes, cafeterías, mercados y aficionados a los festivales (Curiel, 2006)

Una de las ventajas del factor de estudio psicográfico es sencillo de usar ya que las preguntas del cuestionario son comprensivas para cada persona encuestada, además se muestran todos los elementos que se desea analizar sobre el comportamiento del turista que pueden afectar el uso de algún producto o servicio que se desea ofrecer, de la misma manera, da un beneficio a la empresas turísticas o no, a conocer a los consumidores actuales y potenciales de un producto, gracias a esto ayuda a mejorar la promoción a la vez personalizando la oferta que se desea tener, con el objetivo de crear lealtad por parte de los consumidores y así posicionarse en el producto en el mercado (Pérez \& Lois, 2016)

El aspecto socioeconómico analiza el ambiente en el que el turista se encuentra, para conocer cuánto dinero gasta o está dispuesto a gastar durante su visita, además definir los ingresos, nivel de educación y su ocupación laboral porque, mientras tenga mayor poder adquisitivo su interés por conocer nuevos lugares se incrementa, con ello satisfaciendo sus necesidades a la vez llevándose una experiencia agradable, por lo tanto, se refleja en el tipo de productos o servicios que consumen durante su visita (Andrade, 2016). En las características demográficas de los turistas se analizan la edad, genero, características sociales, estado civil y otras variables que permiten identificar la influencia a la hora de adquirir un bien o servicio (Shiffman \& Kanuk, 2005)

\section{La segmentación turística en el mercado}

En turismo la segmentación es importante ya que permite agrupar a los clientes de acuerdo con sus características más comunes en donde el segmento de mercado conforma grupos de consumidores identificando sus deseos, necesidades actitudes y hábitos de compra ante un producto y los más importante con las características del poder adquisitivo, de esta forma ofreciendo a los turistas productos y servicios de calidad satisfaciendo sus necesidades y mejorando sus expectativas de los clientes. Cabe destacar que el objetivo principal de la segmentación de mercados turísticos es acoger a turistas planteando diferentes estrategias atrayentes para el cliente (Klainer, 2003)

\section{Segmentación por el método VALS}

El método Values and Lifestyles VALS, (valores y estilos de vida) creado por Stanford Research Institute (SRI) Internacional S.A fue desarrollado en la década de los ochenta, con un primer formato denominado Values and Lifestyles I (VALS 1) donde agregaba los valores sociales de las personas, combinando datos demográficos, los estilos de vida y los valores, sin embargo, en los años noventa se complementa esta metodología, en base a dos teorías: La 
Teoría de las necesidades jerárquicas de Maslow, y la Teoría de carácter social de Riesman, en ello se trataba de los estilos de vida por las actividades e interés de las personas, en donde se estudia los factores más importantes como el factor psicográfico (Cánovas \& Opazo, 2005)

En la actualidad los estudios de segmentación utilizan el método VALS II, que ayuda a identificar las oportunidades actuales y futuras del mercado consumidor esto se lo hace en base a los rasgos de la personalidad, agrupando agrupar a los grupos de personas según los valores que posee y los estilos de vida que practica. Los valores influyen en las actitudes, conducta y sus necesidades psicológicas, los valores se definen a un consumidor a través del perfil psicográfico porque a la hora de elección las personas adquieren los productos según sus necesidades, opiniones de la familia, preferencias, posicionamiento social y donde habita, este método permite realizar una mejor segmentación del cliente y hacer productos específicos para los diferentes consumidores existentes en los estilos de vida (Salgado \& Camarena, 2014)

Las categorías del sistema VALS II se encuentra desarrollado en base a los estilos de vida, considerando además: a) características personales del consumidor, b) contexto personal, c) las necesidades, estos tres elementos influyen en el diario vivir de cada de unas la personas, concluyendo como administran su dinero y en que lo invierte o gasta además que hacen con su tiempo (Guizar, 2014)

El método VALS II ha tenido una gran acogida siendo esta el más usado para poder ampliar nuevos productos de las empresas siendo estas turísticas o no, creando nuevas estrategias para posicionarse en un mercado a la vez segmentando nuevos mercados metas, este nuevo método tiene 35 ítems con una escala de cuatro puntos; muy en desacuerdo, en desacuerdo, de acuerdo y muy de acuerdo, además, en ella se encuentra 4 preguntas del aspecto demográfico (Salgado \& Camarena, 2014)

En el cuestionario VALS II, cada segmento identificado tiene actitudes de consumidores diferentes, es por eso que este método describe a las personas y su manera de comportarse en grupos, los individuos que adquieren productos o servicios siempre buscan tener experiencias satisfactorias para sus vidas o para su familia (Costa \& Carvalho, 2016)

Existen tres motivaciones que determinan el comportamiento consumidor (Novak \& Bruce, 1990)

- Primarias: determinada por los conocimientos y principios tomando las decisiones basados en ellos mismos.

- Logro: los consumidores buscan encontrar el éxito de los productos o servicios adquiridos para sentirse satisfechos.

- La autoexpresión: experiencias únicas con el producto o servicio

\section{Las dimensiones VALS II}

- Entusiasta: Las personas entusiastas son aquellas que creen en sí mismos, en su capacidad de convertir las cosas, creer en los demás y sobre todo creer en su capacidad de cambiar al mundo y su situación personal, de esta manera dejando a un lado la 
negatividad que piensa con respecto a trasformar y cambiar la situación actual que lo rodea ya que únicamente las personas entusiastas son capaces de dominar los diferentes desafíos u obstáculos que se presenta en el día a día (TemplatesYard, 2018)

- Moda: La moda en el transcurso del tiempo se ha tomado un rol muy importante para las personas ya que es un fenómeno pasajero en un proceso de renovación paulatina, la moda tiene características muy importantes como son la superficialidad, novedad, el gusto por alguna cosa. En la actualidad las personas están interiorizando en los estereotipos de la moda ya sea en la vestimenta, tecnología y en algunas cosas como viajes a lugares diferentes lugares turísticos. (Rojas, 2005)

- Fabricadores: Son aquellas personas que tienen habilidades por lo tanto en su día a día están en una constante innovación de cosas o productos fáciles, además de aquello a estas personas les gusta hacer cosas que pueden utilizar a diario y sobre todo realizarlo con sus propias manos estas pueden ser en materiales tales como de madera, metal u otros (Pérez \& Merino, 2009)

- Líderes: Las personas líderes son aquellas que poseen el poder de influir en los demás, tienen una voz de mando que guía hacia la toma de una buena decisión está siendo de una manera comprensiva, respetuosa a la vez ser líder, pero sin ser un jefe, de esta manera siendo el responsable de un grupo y lo más importante direccionando a otras personas hacia un mejor desarrollo (Gonzalez \& Agudelo, 2014)

- Mecánicos: Son aquellas personas que están interesadas en el funcionamiento de cosas mecánicas tales como motores, maquinas industriales entre otros además de aquello se enfatiza en las personas que les gusta analizar instrumentos de hardware o tiendas automotrices y sobre todo que se refiera a la tecnología (Londoño, 2003)

- Conservadores: Son aquellas personas que cuidan mucho su ideología su pensamiento acerca de la religión, ya que son más enfocadas en creencias divinas y en lo que se refiere a la biblia, además la filosofía de aquellas personas es indispensable en la toma de decisión ya que un acto de mal seria como un acto negativo hacia la persona (Jáuregui, 2015 )

- Alto amor propio : Estas personas tienen características muy peculiares ya que tiene la capacidad de creencia en sí mismo, de esta manera elevando la autoestima (valorándose a sí mismo, amándose, apreciando, y sobre todo aceptando tal como es), además de aquello en esta dimensión la persona se da cuenta de lo que es capaz de hacer como la mayoría de la gente y así pueda tomar decisiones correctas (Pinto, 2002)

- Varios intereses: Esta dimensión se enfoca en las diferentes características de un fenómeno observable de los individuos de una población, de esta manera teniendo en cuenta que cada una de las personas tienen un sinnúmero de necesidades y decesos, además las características de las personas buscan satisfacerlas de acuerdo con las necesidades que poseen. Así mismo hay que tener en cuenta que las personas plantean varios objetivos a cumplir ya sea en lo social, cultural, económico y personal (Colás \& Hernández, 2001)

Una de las ventajas de este sistema es que las empresas lo usan para comprender con mayor facilidad el comportamiento de las personas en el momento de elegir un producto, gracias aquello pueden realizar estrategias para promocionar mejor dentro de un mercado meta, asimismo utilizan este sistema para entender las motivaciones que provocan el comportamiento de las personas, así impidiendo que a las empresas tengan una desventaja con un desorden 
publicitario, las preguntas del cuestionario son sencillas para responder pues miden las motivaciones básicas con esto evitando la menor confusión posible (Mcintyre \& Reid, 1994)

El objetivo primordial del sistema VALS II es de conocer al mercado a la cual está dirigido y lo quiere llegar a ofrecer los productos y servicios turísticos, entre los componentes principales que influyen en el comportamiento de la compra el sistema VALS II determina las siguientes; consumidores orientados por principios, estatus y a la acción, estos ayudando para el éxito de las empresas en desarrollar nuevos productos, innovar estrategias de posicionamiento, segmentar mercado y sobre todo analizar el comportamiento de usuario a la hora de realizar alguna compra (Guizar, 2017)

\section{Segmentación por el método LATIR}

La segmentación estilos de vida Latinoamericanos según actitudes, tendencias, interés y recursos (LATIR) fue iniciado en el año 1996, siendo la primera segmentación original de los consumidores latinoamericanos con valor estadístico y con una proyección continental, la segmentación de este método se ha desarrollado para personas a partir de 18 años, se dio a conocer una vez analizado el pueblo del vecino país del Perú y seguidamente se lo realizo en México, sin embargo en muchos países Latinoamericanos existe diversos tipos de análisis pero coincidiendo en las variables ya que los países tienen una historia y una evolución similar en los últimos 30 años, los estilos de vida identificados en el año 2017 en Perú y en América son 6, para poder identificar los estilos de vida se realiza 12 preguntas sencillas y fácil de responder, esto se analiza con una formula algoritmo que ubica a las personas en su estilo de vida (Arellano, 2017)

Los segmentos identificados por el método LATIR según Rolando Arellano en el año 2017 son las siguientes:

- Sofisticados: es un segmento de genero entre hombres y mujeres, que tienen un ingreso económico alto, además son modernos, educados, liberales y valoran su imagen personal, están pendientes de las ultimas tendencia de la moda, son innovadores al momento de consumir.

- Progresistas: género masculino, normalmente dueños de medianas y pequeñas empresas, le gusta el negocio, estudia carreras técnicas y cortas, no le atrae mucho la moda, le gusta la tecnología y piensa en el futuro de su familia.

- Modernas: género femenino que gusta de una apariencia atractiva, se puede decir que tiene las mismas características del progresista en lo que respecta a trabajo, negocios y emprendimiento.

- Formalistas: está formado por género masculino, se encuentra atrasado en la moda, le gusta ser parte de la sociedad, admira a políticos y personas relevantes, es tradicionalista y tiene creencias muy fuertes sobre religión.

- Conservadoras: se conforma por el género femenino, que no le presta mucha atención a su manera de vestir y maquillarse, salvo para ocasiones especiales, es la ama de casa tradicional que gusta de compartir con sus hijos en el hogar. 
- Austeros: es un género mixto entre hombre y mujeres, se puede decir que rayan la pobreza extrema, viven de lo que venden o trabajan al día, viven en sectores populares, la mayoría de ellos no ha cursado la escuela o apenas han llegado a la primaria.

La segmentación LATIR utiliza datos psicográficos, en su manera de pensar, además, utiliza datos demográficos, económicos también se analiza el comportamiento de las personas, es una herramienta donde permite a las empresas, $\mathrm{ONG}$, asociaciones e instituciones a comprender con mayor facilidad a las personas con las que se vincula día a día (Arellano, 2017)

\section{Materiales y Métodos}

La investigación es cuantitativa, descriptiva y transversal. Se realizó una investigación documental y de campo profunda con la finalidad de obtener la información, utilizando instrumentos de medición validados mediante los métodos VALS II y LATIR que se aplicaron a turistas que visitaron la ciudad de Puyo, siendo estos mayores a 18 años y de ambos géneros, donde se dio a conocer una explicación breve y completa acerca de la encuesta obteniendo un alto grado de respuestas.

El enfoque cuantitativo se basa en la obtención de datos y normalmente se utilizan análisis estadístico, para interpretar los resultados lo que permite aportar al conocimiento científico mediante la comprobación de hipótesis (Sampieri, Collado, \& Baptista, , 2010). El análisis descriptivo fue necesario en la investigación ya que permitió identificar con precisión las características y los perfiles de las personas en los aspectos de atractivos turísticos, alojamiento y restauración quienes visitan la ciudad en los que se pudo medir las variables e identificar los estilos de vida (Sampieri, Collado, \& Baptista, 2010) a.

Se realizó un muestreo estratificado, dividiendo en estratos las actividades de: atractivos turísticos, alojamiento y restauración, para lo cual se aplicó la fórmula estadística de muestreo sin conocer la población, en donde, se obtuvo como requerimiento encuestar a no menos de 384 turistas que visitan la ciudad para obtener una seguridad del $95 \%$.

\section{Resultados}

\section{Variables Demográficas}

Tabla 1.

Género de la muestra

\begin{tabular}{|c|c|c|c|c|c|}
\hline \multicolumn{6}{|c|}{ Genero } \\
\hline & & Frecuencia & Porcentaje & Porcentaje válido & $\%$ acumulado \\
\hline \multicolumn{6}{|l|}{ Válido } \\
\hline & Masculino & 206 & 53,6 & 53,6 & 53,6 \\
\hline & Femenino & 178 & 46,4 & 46,4 & 100,0 \\
\hline & Total & 384 & 100,0 & 100,0 & \\
\hline
\end{tabular}


Tabla 2.

Edad de la muestra

\begin{tabular}{llrrrr}
\hline \multicolumn{7}{c}{ Edad } & & & \\
\hline & & Frecuencia & Porcentaje & Porcentaje válido & \% acumulado \\
\hline Válido & $\mathbf{1 8}-\mathbf{2 5}$ años & 39 & 10,2 & 10,2 & 10,2 \\
& $\mathbf{2 6}-\mathbf{3 5}$ años & 129 & 33,6 & 33,6 & 43,8 \\
& $\mathbf{3 6}-\mathbf{4 5}$ años & 95 & 24,7 & 24,7 & 68,5 \\
& 46-50 años & 67 & 17,4 & 17,4 & 85,9 \\
& $>$ 50 años & 54 & 14,1 & 14,1 & 100,0 \\
& Total & 384 & 100,0 & 100,0 & \\
\hline
\end{tabular}

Tabla 3.

Estado civil

\begin{tabular}{llrrrr}
\hline & \multicolumn{5}{c}{ Estado Civil } \\
\hline & Frecuencia & Porcentaje & Porcentaje válido & \% acumulado \\
\hline & Soltero & 128 & 33,3 & 33,3 & 33,3 \\
Válido & Casado & 177 & 46,1 & 46,1 & 79,4 \\
& Divorciado & 57 & 14,8 & 14,8 & 94,3 \\
& Viudo & 22 & 5,7 & 5,7 & 100 \\
& Total & 384 & 100 & 100 & \\
\hline
\end{tabular}

Tabla 4.

Nacionalidad

Nacionalidad

\begin{tabular}{llrrrr}
\hline & & Frecuencia & Porcentaje & Porcentaje válido & \% acumulado \\
\hline \multirow{3}{*}{ Válido } & Ecuatoriana & 346 & 90,1 & 90,1 & 90,1 \\
& Extranjera & 38 & 9,9 & 9,9 & 100 \\
& Total & 384 & 100 & 100 & \\
\hline
\end{tabular}

Tabla 5.

Cuánto gasta por día

¿Cuánto estaría dispuesto a gastar en la visita por día?

\begin{tabular}{llrrrr}
\hline & \multicolumn{1}{c}{ Frecuencia } & Porcentaje & Porcentaje válido & \% acumulado \\
\hline Válido & $\mathbf{1 0}$-30 USD & 82 & 21,4 & 21,4 & 21,4 \\
& 31-50 USD & 137 & 35,7 & 35,7 & 57,0 \\
& $\mathbf{5 1 - 8 0}$ USD & 90 & 23,4 & 23,4 & 80,5 \\
& $>$ 80 USD & 75 & 19,5 & 19,5 & 100,0 \\
\cline { 2 - 5 } & Total & 384 & 100,0 & 100,0 & \\
\hline
\end{tabular}


Tabla.6

Tipo de comida que prefiere

\begin{tabular}{llrrrr}
\hline \multicolumn{5}{c}{ ¿Qué le gustaría comer durante su visita? } \\
\hline Válido & Frecuencia & Porcentaje & Porcentaje válido & \% acumulado \\
& Comida tradicional & 225 & 58,6 & 58,6 & 58,6 \\
& Comida gourmet & 58 & 15,1 & 15,1 & 73,7 \\
& Comida rápida & 47 & 12,2 & 12,2 & 85,9 \\
Comida light & 19 & 4,9 & 4,9 & 90,9 \\
& Buffet & 35 & 9,1 & 9,1 & 100,0 \\
& Total & 384 & 100,0 & 100,0 & \\
\hline
\end{tabular}

Tabla.7

Tipo alojamiento

\begin{tabular}{llrrrr}
\hline \multicolumn{5}{c}{ ¿Qué tipo de alojamiento desearía? } \\
\hline Válido & Frecuencia & Porcentaje & Porcentaje válido & \% acumulado \\
& Hoteles & 130 & 33,9 & 33,9 & 33,9 \\
& Hostales & 77 & 20,1 & 20,1 & 53,9 \\
& Lodge & 31 & 8,1 & 8,1 & 62,0 \\
& Departamentos & 22 & 5,7 & 5,7 & 67,7 \\
& Cabañas & 124 & 32,3 & 32,3 & 100,0 \\
& Total & 384 & 100,0 & 100,0 & \\
\hline
\end{tabular}

\section{Tabla.8}

Tipo de diversión

¿Qué tipo de diversión le gustaría tener durante su visita?

\begin{tabular}{llrrrr}
\hline & Frecuencia & Porcentaje & Porcentaje válido & \% acumulado \\
\hline Válido & Turismo de Aventura & 126 & 32,8 & 32,8 & 32,8 \\
& Turismo Científico & 33 & 8,6 & 8,6 & 41,4 \\
& Turismo Cultural & 109 & 28,4 & 28,4 & 69,8 \\
& City Tour & 11 & 2,9 & 2,9 & 72,7 \\
& Turismo Gastronómico & 35 & 9,1 & 9,1 & 81,8 \\
& & 13,0 & 13,0 & 94,8 \\
& Turismo de Montaña & 50 & 5,2 & 5,2 & 100,0 \\
Agroturismo & 20 & 100,0 & 100,0 & \\
Total & 384 & & & \\
\hline
\end{tabular}




\section{Variable estilo de vida según modelo LATIR}

\section{Figura 1.}

Tipos de estilo de vida en alojamiento

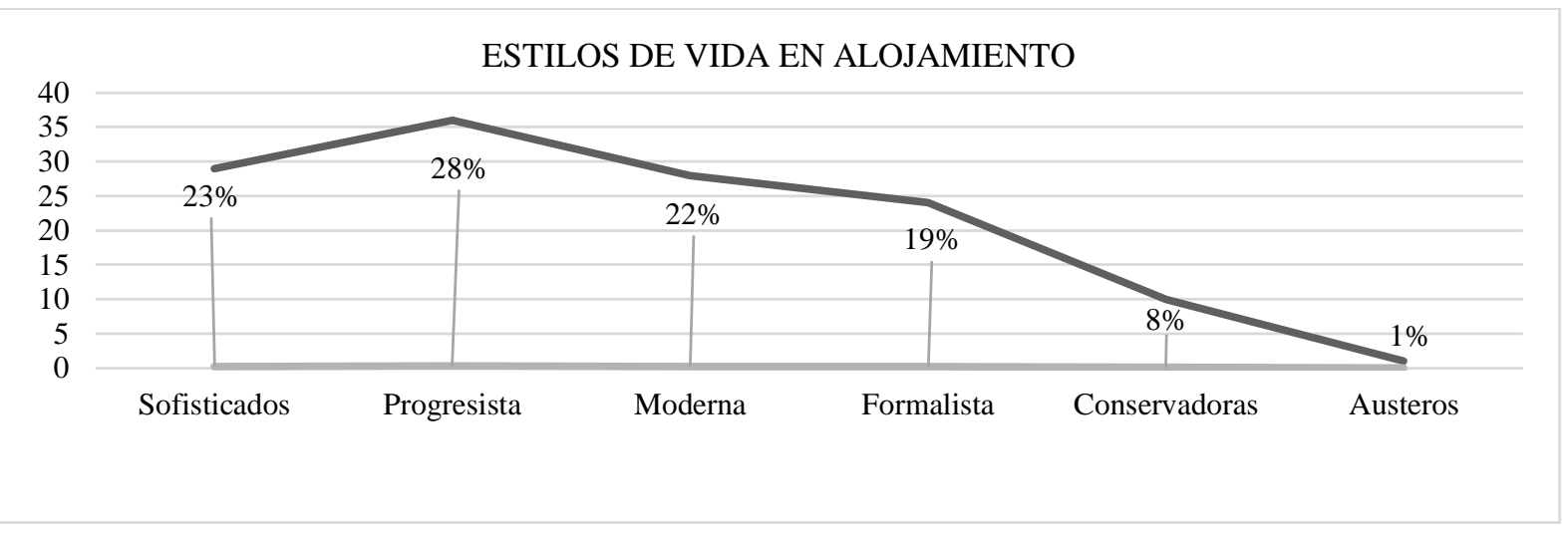

Figura 2.

Tipos de estilo de vida en restauración

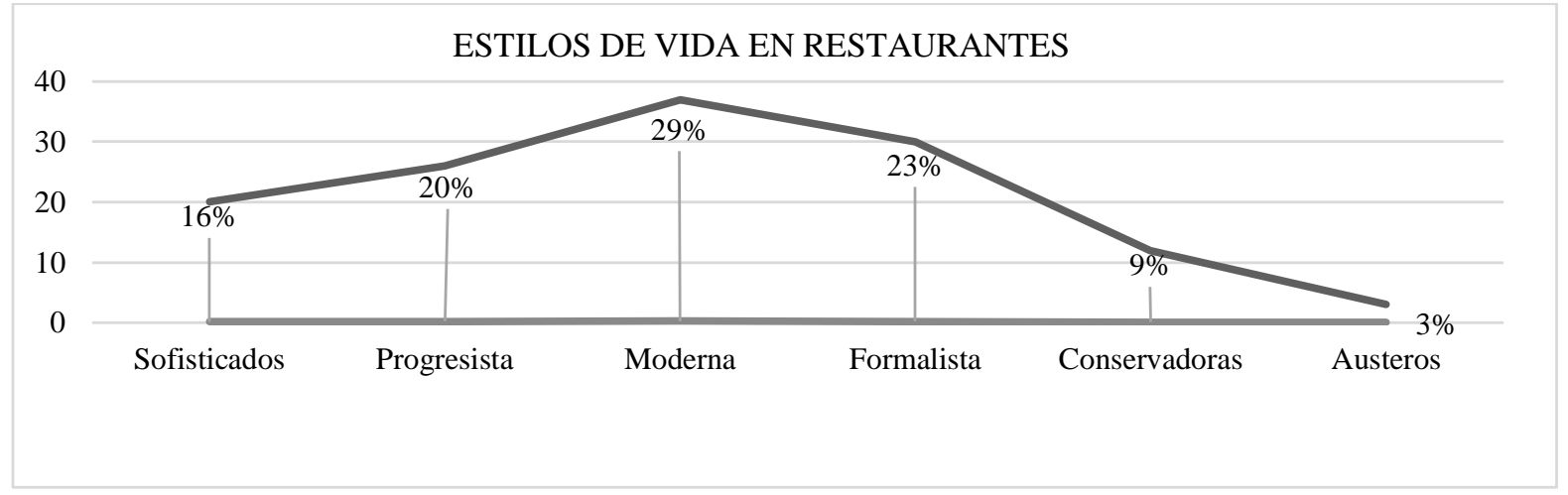

Figura 3.

Tipos de estilo de vida en atractivos turísticos

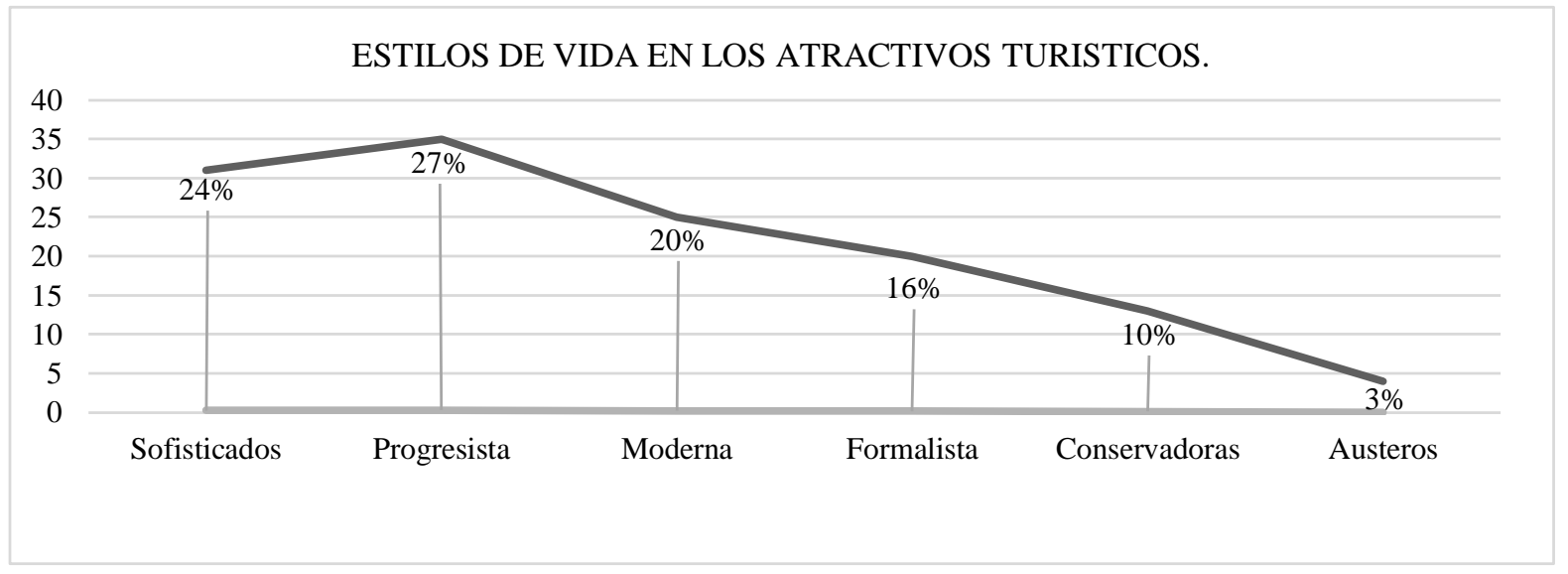

Esta obra se comparte bajo la licencia Creative Common Atribución-No Comercial 4.0 International (CC BY-NC 4.0) Revista de la Universidad Internacional del Ecuador. URL: https://www.uide.edu.ec/ 


\section{Variable valores según modelo VALS II}

\section{Tabla 9.}

Análisis dimensión Entusiastas

Fuente: Elaboración propia

\begin{tabular}{lccc}
\hline & Desfavorable & Poco favorable & Favorable \\
\hline Entusiastas & $0,78 \%$ & $35,94 \%$ & $63,28 \%$ \\
\hline
\end{tabular}

Tabla 10.

Análisis dimensión Moda

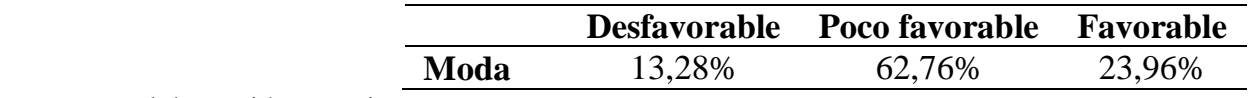

Fuente: Elaboración propia

\section{Tabla 11.}

Análisis dimensión Fabricadores

Fuente: Elaboración propia

\begin{tabular}{lccc}
\hline & Desfavorable & Poco favorable & Favorable \\
\hline Fabricadores & $8,33 \%$ & $55,99 \%$ & $35,68 \%$ \\
\hline
\end{tabular}

Tabla 12.

Análisis dimensión Líderes

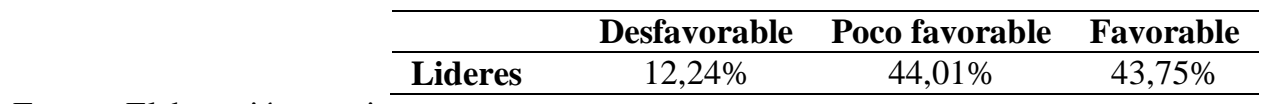

Fuente: Elaboración propia

\section{Tabla 13.}

Análisis dimensión Mecánicos

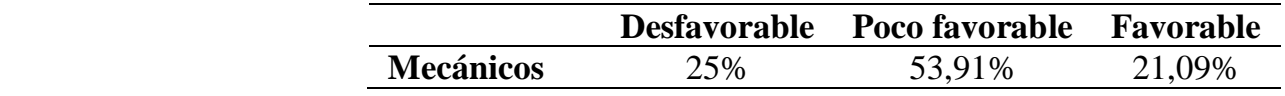

Fuente: Elaboración propia

pia

\section{Tabla 14.}

Análisis dimensión Conservadores

Fuente: Elaboración propia

\begin{tabular}{lccc}
\hline & Desfavorable & Poco favorable & Favorable \\
\hline Conservadores & $14,84 \%$ & $60,94 \%$ & $24,22 \%$ \\
\hline
\end{tabular}

\section{Tabla 15.}

Análisis dimensión Alto amor propio

\begin{tabular}{cccc}
\hline & Desfavorable & Poco favorable & Favorable \\
\hline $\begin{array}{c}\text { Alto amor } \\
\text { propio }\end{array}$ & $6,51 \%$ & $48,70 \%$ & $44,79 \%$ \\
\hline
\end{tabular}


Fuente: Elaboración propia

Tabla 16.

Análisis dimensión Varios intereses

\begin{tabular}{cccc}
\hline Desfavorable & Poco favorable & Favorable \\
\cline { 2 - 3 } & $0,26 \%$ & $67,97 \%$ & $31,77 \%$ \\
\hline
\end{tabular}

Fuente: Elaboración propia

\section{Conclusiones}

Según los datos demográficos analizados se pudo identificar que la mayor cantidad de visitantes son del género masculino en comparación del género femenino, la edad que predomina va entre los 26 años hasta 50 años, la mayor parte de turistas son casados es decir vienen en compañía de su familia y el resto de visitantes son personas solteras quienes vienen acompañados con sus amigos, giras de colegio o universidades, en su mayoría son de nacionalidad ecuatoriana, además arriban turistas extranjeros provenientes de Colombia , Venezuela, Argentina, México y Perú.

Los turistas quienes visitan la ciudad están dispuestos a gastar en un día de visita hasta 80 dólares americanos ya sea en alimentación, recreación y hospedaje, además una de sus mayores expectativas a la hora de degustar un alimento es la comida tradicional entre ellas destacándose los platos a base de tilapia ( Oreachromis niloticus) como en su forma tradicional se conoce como "maito de tilapia", jugo de guayusa ( Ilex guayusa), chontacuro ( Rhynchophorus palmarum)en diversas preparaciones, así mismo a hora de pernoctar prefieren los hoteles y cabañas, las actividades que le gusta practicar son el turismo de aventura, turismo cultural y el turismo de montaña.

Los resultados indicaron que el estilo de vida de los turistas que vistan la región amazónica, específicamente la ciudad de Puyo en su mayor parte, corresponde a un segmento por estilo de vida Progresistas, Modernas y Sofisticados para los establecimientos de alojamiento restauración y atractivos turísticos. Mediante el instrumento de medición VALS II (medida de valores) se pudo identificar que los visitantes son entusiastas, es decir, los visitantes tienen un nivel de ánimo elevado y con muchas expectativas en realizar actividades, sin embargo, en lo referente a las dimensiones moda, fabricante, líder, mecánico, conservador, alto amor propio, se obtuvieron respuestas que van desde poco favorable a desfavorable, esto puede dar lugar a futuras investigaciones para evaluar qué tipo de valores son los que más se identifica en cada uno de los estilos de vida, de la misma manera, se abren nuevas líneas de investigación para identificar causalidades entre lo que valoran los turistas y el estilo de vida que llevan.

\section{Bibliografía}

Andrade, T. (2016). Perfil del turista que visita la ciudad de Cuenca,influido por su reconocimiento como ciudad patrimonio de la humanidad. Universidad de Cuenca, 100.

Angelucci, L., Cañoto, Y., \& Hernández, M. (2017). Influencia del estilo de vida, el sexo, la edad y el imc sobre la salud física y psicológica en jóvenes universitarios. urosario.edu.com. 
Arellano, R. (2017). Estilos de vida LATIR. En R. Arellano, Mucho más que tener: LATIR,Los estilos de vida Latinoamericanos según actitudes, tendencias intereses y recurso (págs. 20-21). Lima: Planeta Perú S.A.

Arellano, R. (2017). LATIR es una forma de segmentación psicográfica. En R. Arellano, Mucho más que tener: LATIR, Los estilos de vida Latinoamericanos según Actitudes, Tendencias, Interes y Recursos. (págs. 27-30). Lima: Planeta S.A.

Arellano, R. (2017). Los estilos de vida de los Latinoamericanos de hoy. En R. Arellano, Los estilos de vida Latinoamericanos segun Actitudes, Tendencias, Intereses y Recursos. (págs. 15-18). Lima: Planeta Perú S.A.

Baudrillard, J. (1970). La Societé de Consommation. Paris:Gallimard, 8.

Boote, A. (1982). Psychographic Segmentation inEurope. Journal of Advertising Research.

Campo, L., Beltran, Y., \& Puello, F. (2017). Estilos de vida saluadables de niños, niñas y adolescentes. Cielo, 2.

Cánovas, A., \& Opazo, C. (2005). Los Segmentos VALS II. Segmentación Psicográfica: 'Una aplicación para Chile'., 89-92.

Cánovas, A., \& Opazo, C. (2005). Segmentación Psicográfica:" Una aplicación para Chile". Universidad de Chile. Facultad de Ciencias Económicas y Administrativas., 38.

Castillo, E., Martínez, F., \& Vazquez, E. (2015). El turismo en Ecuador, nuevas tendencias en el turismo sostenible y contribucción al crecimiento económico. Galega de Economía , 4.

Ciribeli, P., \& Miquelito, S. (2015). La segmentación del mercado por criterio psocográfico: Un ensayo teórico sobre los principales enfoques Psicográficos y su relacion con los criterios de comportamiento. Visión de Futuro, 33-50.

Colás, L., \& Hernández, F. (2001). Métodos de la investigación en Psicopedagogia. McGraw-Hil, 4.

Cordente, M. (2011). Medición de motivaciones del turismo cultural en ciudades. Análisis turísticos, 80-85.

Curiel, E. (2006). La demanda del turismo rural y vinculación con el medio ambiente urbano: los casos de Madrid y Valencia. Universidad Complutense de Mdrid, Facultad Ciencias Políticas y Sociología.

Fernandez, A., Blanco, M., \& Martinez, N. (2013). La incidencia de los estilos de vida en la segmantacion del mercado turistico;aplicaccion a la poblacional urbana de Castilla y Leon. Dialnet, 5.

Fernández, G., Morlegan, C., \& Ramos, A. (2007). Alojamientos Turisticos y Medio Ambiente. Diagnostico Ambiental de los Complejos de Cabañas en Tandil. Argentina . Electrónica $U A C H, 3$.

Gonzalez, D., \& Agudelo, J. (2014). LIDERAZGO: DESARROLLO DEL CONCEPTO, EVOLUCIÓN Y TENDENCIAS. Universidad del Rosario , 15.

Guizar, O. (2014). El sistema VALS. Universidad Virtual del Estado de Micheacán, 23.

Guizar, O. (2017). Sistema de segmentación VALS. Mercadotecnia Turistica, 2.

Jáuregui, M. (2015 ). "El pensamiento conservador en la Constitución de 1980". Universidad de Chile, 9.

Klainer, V. (2003). Segmentación psicográfica, conocer al consumidor. Instituto Tecnológico Autónomo de Mexico., 13.

Kotler, P., \& Keller, K. (2012). Dirección de Marketing. Pearson Educación, 808.

Lazer, W. (1963). "Life - Style Concepts and Marketing". Coference of America Marketing Association: Towar Scientific Marketing,Fall , 130- 139. 
Londoño, M. (2003). Introducción a la Mecánica . Universidad Nacional de Colombia , 8.

López, V., Espíndola, F., Calles, J., \& Ulloa, J. (2013). Atlas "Amazonía Ecuatoriana Bajo Presión". EcoCiencia. Quito-Ecuador., 7.

López, X., \& Martin, B. (2016 ). Restauración Local y Poductos Alimentarios. La situacion en la comarca del Moianés. Cataluña. Revista de Estudios sobre Despoblación y Desarollo Rural , 2-3.

Mcintyre, R., \& Reid, C. (1994). "Empirical relationships between Cognitive Style and LOV: Implications for Values and Value Systems. Advances in Consumer Research, 14.

Morgan, C., \& Levy, D. (2003). Psychographic Segmetation:How to increase ROI by examing values,beliefs and motivations. Communicatios World, 32.

Nieto, J., Róman, I., Bonillo, D., \& Paulova, N. (2016). El turismo a nivel mundial. International Journal of Scientific Management and Tourism, 1-2.

Novak, T., \& Bruce, M. (1990). On comparing alternative Sementation Shemes: The list of Vlues and values and Styles VALS. Journal of Consumer Research, 105.

Pérez, Y., \& Lois, R. (2016). El perfil del visitante en Santiago de Compostela: Tradición y Actualidad. Cuadernos de Turismo, 5.

Pinto, B. (2002). Psicología del Amor. Universidad Católica Bolivariana San Pablo , 10-11.

Reyes, V., \& Ortega, F. (2013). Potencial turístico del Circuito Vial Puyo - Fátima - 10 de Agosto, en la Provincia de Pastaza - Ecuador. Amazonica, 1.

Rojas, N. (2005). La Moda y la Comunicación . Universidad Abierta Interamericana, 7-8.

Salgado, L., \& Caremena, D. (2015). Los valores y estilos de vida de los jóvenes como factores de influencia en el consumo de alimentos internacionales. Poliantea, 5.

Sampieri, R., Collado, C., \& Baptista, M. (2010). Metodología de la Investigación . México : McGraw-Hill Companies .

Sampieri, R., Collado, C., \& Baptista, P. (2010). ¿En qué consisten los estudios de alcance descriptivo? En R. Sampieri, C. Collado, \& P. Baptista, Metodología de la investigación. (pág. 80). México: Interamericana Editores S.A.

Shiffman, G., \& Kanuk, L. (2005). Comportamiento del consumidor. Prentice Hall Mexico, 20.

TemplatesYard. (15 de 04 de 2018). Cultura al Polo. Obtenido de Cultura al Polo : https://culturaalpalo.blogspot.com/2018/04/ser-entusiasta-como-forma-de-vida.html 